\title{
La gestión integrada del conocimiento del consumidor. Caso aplicado a la Planificación Estratégica en España
}

Cristina Sánchez Blanco | csblanco@unav.es

UNIVERSIDAD DE NAVARRA

Resumen: Se analiza la importancia del consumidor en comunicación comercial y de su conocimiento para obtener una comunicación eficaz y su fidelidad. Para ello, ese conocimiento debe ser transversal y los diferentes profesionales implicados deben trabajar de forma coordinada. En publicidad, los planificadores estratégicos son los representantes del consumidor mediante la investigación y la gestión del conocimiento. La revisión bibliográfica se ha aplicado al análisis de la realidad de los planificadores estratégicos españoles. Se hace hincapié en su relación con otros actores del proceso de comunicación comercial para descubrir si se da una gestión integrada del conocimiento del consumidor.

Palabras clave: consumidor, conocimiento, planificación estratégica, integración.

Abstract: This article discusses the importance of consumer in advertising and the consumer knowledge for effective communication and his loyalty. That knowledge must be transverse and the different professionals must work in harmony. In advertising, strategic planners are consumer representatives through research and knowledge management. The literature review has been applied to the analysis of the reality of strategic planners in Spain. This study emphasizes their relationship with others in the business communication process to discover if there is an integrated knowledge management of the consumer.

Key words: consumer, knowledge, strategic planning, integration. 


\section{Introducción}

El consumidor debe ser el centro de los esfuerzos comunicativos de cualquier empresa y, para conseguir la eficacia y la rentabilidad, debe saber cómo es su consumidor y actuar en consecuencia.

El primer objetivo de este articulo es hacer una revisión bibliográfica para demostrar la exigencia, más que necesidad, de conocer al consumidor en profundidad y de saber gestionar ese conocimiento de una manera integrada para sea compartido entre cualquier profesional de la comunicación comercial. El trabajo por el conocimiento del consumidor se concreta actualmente en la figura de planificadores estratégicos en las agencias de publicidad y cada vez más en agencias de medios, anunciantes y otras empresas especializadas, quienes velan por tener un conocimiento de los consumidores lo más profundo posible que pueda ayudar al anunciante.

De esta manera, en la segunda parte de este texto se intentará entender, desde el punto de vista de los profesionales responsables del consumidor, los planificadores estratégicos, si en el proceso de comunicación comercial hay una gestión integrada del conocimiento del consumidor. Se pretende hacer un acercamiento a esta realidad desde el punto de vista de los planificadores, los que más trabajan de cara al consumidor, a los que se ha encuestado para ver qué relación tienen con otros miembros de las agencias de publicidad (cuentas y creativos), con agencias de medios, e institutos de investigación, para ver si con su trabajo ayudan a que el consumidor esté realmente en el centro.

\section{Importancia del conocimiento del consumidor y de la gestión de dicho conocimiento}

Hoy en día, la importancia del producto se ha sustituido por la del consumidor. Esto quiere decir que la información sobre el consumidor es una ventaja competitiva de las empresas porque el nuevo mercado está dirigido por la comprensión del consumidor, el desarrollo de proposiciones comunicativas que le aporten valor, y que sean más eficientes que las de la competencia (Kitchen, Schultz, 2000: 18).

Por ello, la publicidad ahora no se centra en factores como alcance (cuántos consumidores ven un anuncio) o frecuencia (cuántas veces lo ven) sino en encontrar maneras de que las marcas formen parte de la vida de los consumidores (Pérez Latre, 2007: 44). Con la proliferación de medios digitales cobran importancia otro tipo de medidas, como son, entre otras: el tiempo de sesión, el tráfico a la Web, la atención activa al contenido, los clicks, las descargas, la participación de los consumidores, etc. (Vollmer, Precourt, 2008: 113).

Con todo, se entiende que la publicidad de masas va perdiendo sentido y Grant habla de un nuevo marketing más humanista que exige una necesidad de fomentar una relación con las personas y de construir las marcas de cara a ellos (Grant, 2000: XIII-XVI). 
Es evidente que trabajar poniendo al consumidor en el centro es más efectivo (Humby, 2004: 119). En este contexto, es necesario que haya una nueva comunicación de marketing para este nuevo consumidor (Van Raaij, 1998: 7). Rapp y Collins defienden un "gran giro del marketing" que se concreta en que es un paso de consumidores y clientes desconocidos a consumidores y clientes conocidos, de la creatividad como la fuerza impulsora a la respuesta del consumidor como centro, de números de impactos publicitarios a nuevos clientes ganados, de monólogo publicitario a diálogo con el consumidor, de bombardear el mercado a establecer relaciones y de consumidores pasivos a participantes involucrados (1991: 37). Gracias a esta reflexión, se entresacan las ideas más importantes que nos van a servir de base para analizar cómo las empresas están trabajando para que sus acciones estén centradas en el consumidor: conocimiento, respuesta, fidelización, diálogo, relación y participación.

Estas ideas se van a desarrollar a partir de dos grandes puntos: la necesidad de obtener un conocimiento profundo sobre el consumidor y la centralidad del consumidor como resultado de una gestión integrada de su conocimiento.

\subsection{Conocimiento profundo del consumidor}

Aunque se sigue dando una cierta relevancia a la publicidad tradicional, "ahora el lugar principal del pensamiento estratégico lo ocupa la relación deseada con los consumidores individuales" (Rapp, Collins, 1991: XXI). Por ello, se trata de alcanzar al consumidor de una manera nueva porque las que había hasta ahora no son del todo eficaces. García llama la atención acerca de una "Nueva Publicidad" y defiende que "la involucración del consumidor es la base sobre la que se construyen los nuevos modelos de publicidad" (García, 2007: 75).

Las empresas tienen que asegurar que los consumidores están incorporados en el proceso de marketing mediante un diálogo, que gracias a la tecnología interactiva, posibilita tener con cada consumidor, de una manera barata y sostenible a largo plazo. Así, se intentará conseguir su lealtad (Godin, 2000: 12-13). Pero, para poder desarrollar acciones para los consumidores, el primer paso es hacerse cargo de las necesidades y del contexto de esas necesidades para conectar con ellos y poder diferenciarse de la competencia (Fauconnier, 2006: 37).

Esta situación tiene consecuencias para los profesionales del marketing, las agencias y los medios. Entender al consumidor en este contexto es más importante que nunca (Considine, 2007: 24) ya que el comportamiento del consumidor es la clave para desarrollar la estrategia de publicidad. Los anunciantes se gastan mucho dinero en que los individuos se interesen por sus productos. Para tener éxito, tienen que saber por qué se comportan de una determinada manera (Arens, Schaefer, 2007: 126-127). Se trata de entenderles por completo para saber qué relación tienen con la marca, cuáles son sus intereses, sus 
preferencias o sus hábitos de compra y desarrollar una segmentación de mercado eficaz para alcanzar a los públicos objetivos (Yeshin, 1998: 11).

Se necesita desarrollar un sistema que recoja toda la información de todos los puntos de contacto (Swain, 2004: 48) del consumidor, esto es, de cualquier experiencia en la búsqueda de información que el consumidor tiene con la marca. Hay muchas maneras de que una persona se acerque a una marca: los amigos, el trato en el punto de venta, el packaging, la televisión, etc. Y los contactos no terminan con la compra (Schultz, Tannenbaum, Lauterborn, 1996: 47-51), sino que también es necesario potenciar la relación posterior para facilitar la fidelización de los consumidores.

La comunicación integrada es la clave para que los valores de las marcas, de los productos y de los servicios sean comunicados al consumidor en todos los canales accesibles y asegurar que la marca mantiene una posición fuerte en la mente del consumidor. A largo plazo, las marcas sólo pueden competir si usan todo el potencial de opciones de comunicación que tienen al alcance para llegar al consumidor (Franz, 2000: 42). Pero la integración tiene que estar bien entendida: no es unir diferentes medios para dar un servicio multimedia al consumidor sino poner el foco en el consumidor y pensar cómo involucrarle a través de cualquier punto de contacto (García, 2007: 129) y emplear los medios necesarios para encontrar al consumidor deseado.

\subsection{La centralidad del consumidor como resultado de una gestión integrada del conocimiento del consumidor}

Un elemento interesante para gestionar el conocimiento del consumidor son las bases de datos y en concreto los sistemas Customer Relationship Management (CRM). Sin embargo, Chiesa incide en la idea de que no sirve de nada desarrollar una herramienta tecnológica muy interesante si no se analizan los datos que se obtienen, se interpretan y se toman decisiones para alcanzar las metas previstas (2005: 232-233).

Esta idea también la defienden Rapp y Collins cuando afirman que el desarrollo de una base de datos por sí solo no soluciona nada. Es solamente el medio para llegar a un fin y ese fin es el contacto, el diálogo y la relación directa con el consumidor o cliente individual que dará lugar a un incremento en las ventas y en la lealtad a la marca (1991: XXII). No hay que confundir contar con muchos datos de los consumidores con entenderlos realmente.

Por ello, hay que pasar de una simple gestión de bases de datos a una gestión integrada del conocimiento del consumidor, que se basa en una verdadera y profunda relación con los consumidores que sirva para orientar la estrategia y la política comunicativa de las empresas. Éste es el principio del marketing uno a uno, cuyo fin es dar valor a la relación con el consumidor (Newell, 2000: 2). 
Así, se entiende que lo más importante es conocer a fondo a los consumidores "para elaborar una estrategia de marketing válida" (Zaltman, 2004: 51). Los consumidores piden que se les respete como personas individuales, no como simples datos demográficos y que se les ayude a hacer unas buenas elecciones de marca. El éxito de una empresa depende de esta lealtad de los consumidores (Sirkin, 2004: 87). Lo importante en la gestión de los consumidores no son los datos únicamente sino saber acceder a ellos (Calder, 2001: 163-164) y convertirlos en los "héroes" de la compañía (Rensmann, 2000: 18).

Y este conocimiento profundo que se obtiene debe ser propiedad de toda la empresa y debe estar correctamente gestionado de una manera integrada por todos los participantes del proceso de comunicación comercial, esto es, por las agencias de publicidad, los anunciantes, los institutos de investigación y agencias de medios como actores más destacados. Esto quiere decir que, para que el consumidor ocupe un lugar central en la comunicación y eso repercuta en su eficacia debe ser el protagonista de todas las etapas del proceso que no deben trabajar de forma independiente sino integradas porque se trata del mismo consumidor. Así, el objetivo que tiene el anunciante de conectar con el consumidor de una manera eficaz y conseguir su satisfacción y su compromiso pasa por desarrollar una campaña de comunicación que le atraiga y situar el mensaje en unos medios donde el consumidor se encuentre. Si todos los profesionales tienen al consumidor como centro y fin de sus acciones, esta meta se conseguirá más fácilmente.

De esta manera se trabaja para buscar el compromiso del consumidor y Chiesa considera que la fidelización de los clientes "debe ser el objetivo final de un nuevo planteamiento de marketing, a fin de poder permitir a las empresas ser consideradas excelentes en el mercado actual. Esto significa que el marketing moderno no puede tener como único objetivo el lograr una venta, sino iniciar una relación" (2002: 37).

Los profesionales del marketing en cualquier ámbito o estadio del proceso de comunicación comercial (agencia de publicidad, de medios o de investigación) deben identificar, aprender, trabajar y estar pendientes de los consumidores todo el tiempo y a todos los niveles (Schultz, Tannenbaum, Lauterborn, 1994: 178), estar relacionados, más bien involucrados, con la historia y la vida de sus consumidores (Duncan, 2005: 90) y potenciar una verdadera relación con ellos. Estableciendo esta relación, las compañías han encontrado estabilidad en un mundo de ciclos de productos rápidos e innovación y competencia constante (Rensmann, 2000: 17).

Para que todo esto se dé en la realidad y el consumidor efectivamente esté en el centro del proceso de comunicación comercial, es necesario que existan profesionales cuyo objetivo principal sea velar por conocer al consumidor y por gestionar ese conocimiento. Estos son los planificadores estratégicos que, con su trabajo, hacen ver que el consumidor debe ser el centro de todo el proceso. 


\section{Aplicación al caso de los planificadores estratégicos}

3.1. Los planificadores estratégicos, responsables de la gestión integrada del conocimiento del consumidor

En el origen de la Planificación Estratégica se reconocen dos escuelas (O’Malley, 1999: 43), dos visiones de la misma realidad, lideradas por Stephen King y Stanley Pollitt. Ambos personajes, con trayectorias profesionales diferentes (King trabajó en la agencia J. Walter Thompson y Pollitt en Pritchard Wood Partners), compartían un interés común: lograr el conocimiento del consumidor y volcarlo en la elaboración de anuncios para mejorar la eficacia de la publicidad. La Planificación Estratégica nació, en este sentido, en el entorno de las agencias de publicidad con el objetivo de que una persona preparada se ocupara de la investigación en profundidad sobre el consumidor para integrar ese conocimiento en toda la agencia. Así, el planificador era la conciencia del director de la cuenta y cooperaba con el trabajo de los creativos para obtener una publicidad eficaz (Sánchez Blanco, 2009: 199).

Tras más de cuarenta años de evolución, en la actualidad, el planificador trabaja en el análisis de mercado y del consumidor, en el desarrollo de la publicidad y de la marca, en la evaluación, y en conocer las posibilidades de cada disciplina de comunicación de marketing (Baskin, Pickton, 2003: 421) para poder integrar ese conocimiento en todo el proceso de comunicación comercial.

En concreto, con el desarrollo de la sociedad de la información y la fragmentación de los medios, los planificadores han pasado a ser planificadores de comunicación. Coordinan estratégicamente la marca a través de los diferentes tipos de comunicación de marketing y conocen todas las habilidades de las diferentes disciplinas y cómo se accede al consumidor a través de ellas. En este entorno, se entiende que el papel del planificador sea indispensable porque controla la visión central de la marca que se tiene que transmitir en cada tipo de comunicación. Los planificadores, así, se encargan de la comunicación total de la marca ya que separar la creatividad y los medios no es necesario ni deseable. Ambos aspectos dependen de entender al mismo consumidor. Por ello, hoy en día las empresas demandan un perfil que tenga habilidades de planificación de medios y de planificación de la marca. Así, vemos cómo el planificador estratégico tradicional se convierte en un brand/media/communications planner (Zambardino, Goodfellow, 2003: 432).

Gracias al origen de la Planificación Estratégica en la publicidad se dio relevancia a las personas, a los consumidores. $\mathrm{Y}$ esto ha ido madurando, a través de la integración en los años 90, hasta llegar a la actualidad donde lo fundamental es el modo en el que las marcas se relacionan con las vidas de sus consumidores. Los planificadores estratégicos colaboraron en el desarrollo humanístico de la publicidad en los años 70, y son claramente capaces de hacer lo mismo por los medios hoy; esto es, poner al consumidor en el centro de la comunicación, reclamando su territorio de grandes estrategas de consumidores (Kaye, 
2002). El planificador estratégico es quien trabaja para gestionar de manera integrada el conocimiento del consumidor y quien es capaz de hacer entender al anunciante que, si quiere ser eficaz a largo plazo, tiene que trabajar para y con el consumidor.

En este sentido, es interesante analizar qué papel juega el consumidor en el proceso de comunicación comercial, desde el punto de vista del profesional que realmente siente al consumidor como su responsabilidad. Así, se realizó una encuesta a los planificadores estratégicos que trabajan en España a los que se les preguntó qué relación tienen con otros participantes del proceso de comunicación como creativos y ejecutivos de cuentas de las agencias de publicidad y otras empresas como agencias de medios, institutos de investigación o los mismos anunciantes y qué relevancia dan estos profesionales al consumidor y a su conocimiento. Así, se podrá ver si, en la opinión de los planificadores estratégicos, existe actualmente una gestión integrada del conocimiento del consumidor en la comunicación comercial.

\subsection{Aplicación al caso español. Universo y muestra}

El universo objeto de estudio eran todos los planners ${ }^{1}$ que trabajan en España. Gracias a la información que facilitó la Asociación de Planificación Estratégica de España (APG España), conviene señalar que no hay ningún censo oficial que contenga el número de profesionales de este campo. Además, este análisis, realizado a finales de 2008, presentaba otra dificultad porque esta disciplina es complicada de definir, entre otras, por estas circunstancias:

Hay agencias que, desde otros departamentos, ejercen las funciones de planificador estratégico pero sin denominarlo así. Esto se puede dar, porque, en el fondo, lo importante es que cale la filosofía, que se lleven a cabo las tareas anteriormente mencionadas y que se vele por los intereses del consumidor, sin tener sólo en cuenta el departamento que lo desarrolle.

Hay planners que trabajan de manera freelance.

Como se ha visto, cada vez más, hay otras empresas que tienen planners, no sólo agencias de publicidad convencional, sino también de medios no convencionales, consultoras de marcas, agencias de medios, etc.

Desde APG España, instituida en 2006 y en la actualidad con aproximadamente 150 miembros, destacan que no todos son planners activos sino que hay un gran grupo de otros profesionales de agencias (del departamento de Cuentas y de Creatividad), académicos, estudiantes y otros profesionales que aún no han encontrado trabajo como planner. En este sentido, es necesario resaltar que, por tanto, el número de planificadores objeto del estudio

\footnotetext{
${ }^{1}$ En la profesión se habla de planificadores o planners, así que se empleará indistintamente los dos términos.
} 
era menor, pero en contraposición también se procuró llegar a otros que no fueran socios. Finalmente, se recibieron 62 respuestas, 50 miembros de la asociación, por lo que, por lo menos, están representados más de un tercio de los miembros.

Según el ranking de agencias por inversión gestionada, que realiza Infoadex ${ }^{2}$, se demuestra que, de las 16 primeras agencias, han contestado 14, lo que corresponde al 87,5\% de las agencias de publicidad más importantes en España, de lo que se deduce que las mejores agencias españolas tienen planificadores estratégicos.

Por otro lado, se ha contado con una gran representación de otras agencias de publicidad más pequeñas que también aportan conclusiones muy importantes. Además, el estudio se ha visto enriquecido con las opiniones de tres consultoras de marca o de marketing, Added Value, Futurebrand y WINC, cuatro agencias de medios, CIMC, Initiative, Arena Media Communications y Carat/Aegis Group, un responsable de marketing y comunicación del anunciante y dos freelance.

Vemos a continuación un gráfico que muestra qué empresas han participado en el estudio.

\begin{tabular}{|c|c|}
\hline Nombre Agencia & Respuesta \\
\hline MCCANN-ERICKSON & 4 \\
\hline BASSAT OGILVY \& MATHER & 1 (OgilvyOne Worldwide) \\
\hline DDB & 2 \\
\hline PUBLICS ESPAÑA & 2 de Publicis España y 2 de Publicis Dialog \\
\hline GREY & 4 \\
\hline TAPSA & 2 \\
\hline EURO RSCG ESPAÑA & 1 \\
\hline SEÑORA RUSHMORE & Ninguna \\
\hline TBWA/ESPAÑA & 3 (1 de ADDING TARGIS (TBWA WorldHealth) \\
\hline YOUNG \& RUBICAM & 5 (3 de Vinizius) \\
\hline VITRUVIO LEO BURNETT & 1 \\
\hline CONTRAPUNTO & Ninguna \\
\hline TIEMPO BBDO & 1 \\
\hline DEVILCO & 1 \\
\hline SAATCHI \& SAATCHI & 1 \\
\hline
\end{tabular}

2 Publicado por Anuncios el 28 de septiembre de 2008. Disponible en http://www.anuncios.com/ inversion-publicitaria/mas-anuncios/1028854009501/media-planning-mccann-erickson.1.html (consultado: 29/09/08). 


\begin{tabular}{|c|c|}
\hline JWT SPAIN & 1 \\
\hline AZK & 2 \\
\hline BRAINSTANSOUP & 1 \\
\hline DOMMO CREATIVE CENTER & 1 \\
\hline FMRG COMPACT & 1 \\
\hline FUTUREBRAND & 1 \\
\hline GLOBAL HEALTHCARE BARCELONA & 1 \\
\hline GREAT WORKS & 1 \\
\hline HERRAIZ SOTO \& CO & 1 \\
\hline MULTIPLICA & 1 \\
\hline ORBITAL & 1 \\
\hline PRISMA GRUPO & 1 \\
\hline SHACKLETON & 2 \\
\hline TÁCTICA INVESTIGACIÓN Y ESTRATEGIA & 1 \\
\hline VIDALLARSSON-DUPREZ & 1 \\
\hline WYSIWYG & 2 \\
\hline LOLA & 1 \\
\hline DEC & 1 \\
\hline ARENA MEDIA COMMUNICATIONS & 1 \\
\hline CARAT/AEGIS GROUP & 1 \\
\hline CICM & 1 \\
\hline INITIATIVE & 1 \\
\hline FREELANCE & 2 \\
\hline MARKETING ANUNCIANTE & 1 \\
\hline $\begin{array}{l}\text { WINC, consultora de marketing y } \\
\text { comunicación }\end{array}$ & 1 \\
\hline Marketing Jazz, punto de venta & 1 \\
\hline ADDED VALUE & 1 \\
\hline
\end{tabular}

Gráfico 1. Elaboración propia con datos del estudio. 


\subsection{Metodología: encuesta}

La Planificación Estratégica es una disciplina bastante reciente en España (Ollé, 2005: 116) y no hay un análisis de su situación. Así, se intentó llegar al mayor número posible de planners. De esta manera, se escogió una encuesta con cuestionario autoadministrado (Pedret, Sagnier y Camp, 2000: 196).

El cuestionario, con un total de 51 preguntas, de las cuales la mayoría, 40, son cerradas, 8 semicerradas y 3 abiertas, fue elaborado durante dos meses y pasó por diversos filtros y correcciones de profesores con experiencia en la elaboración de este tipo de investigación. Constaba de tres grandes apartados:

1. Perfil de los participantes

2. Trabajo diario del planificador estratégico:

a. Visión estratégica

b. Investigación sobre el consumidor y gestión del conocimiento del consumidor

c. La Planificación Estratégica, disciplina flexible

3. Relación del planificador con los actores de comunicación comercial

Para la elaboración de este artículo se ha sacado la información más relevante, del punto b y del apartado 3.

Antes de enviarlo a los profesionales se testó mandándolo a varios planners para retocar aspectos formales y de contenido, que mejoraron en gran medida el resultado final. Como la muestra era amplia se decidió trabajar con el cuestionario de manera online. Así, se envió un mail con el enlace de la página web donde fue publicado el cuestionario. Esto presentaba varias ventajas:

1. Para el encuestado resultaba fácil acceder al enlace desde su correo electrónico y al acabarlo únicamente tenía que pulsar "enviar cuestionario", por lo que no era necesario contestar el mail.

2. El investigador podía acceder a los datos de una manera sencilla. Después de realizar las sintaxis pertinentes se obtuvieron los datos a través del programa SPSS, con el que pudimos realizar el análisis de forma muy completa. 
Para acceder a la muestra se dieron varios pasos. El más importante fue el apoyo y la colaboración de la APG España, desde cuya secretaría se envió el cuestionario por mail a todos los socios. En segundo lugar, se empleó un método no probabilístico denominado muestreo por bola de nieve que, como explica Fernández Nogales:

Consiste en solicitar de las propias unidades muestrales captadas la identificación de posibles nuevos elementos de la muestra pertenecientes al colectivo objetivo. Este procedimiento es apropiado para poblaciones reducidas y muy especializadas que presentan dificultades para su identificación (1999: 131).

3.4. Resultados: relación del planificador con los participantes del proceso de comunicación comercial

Si el consumidor es el centro de la comunicación de marketing y el planner es el que le conoce en profundidad, en el entorno actual parece que cobra más importancia que se dé una gestión integrada del conocimiento del consumidor y que sea el planner el que la lidere. En este sentido, es indispensable que los participantes del proceso de comunicación comercial tengan la misma información y trabajen poniendo el foco en el consumidor. De esta manera, en este estudio empírico se analizan, siempre desde el punto de vista del planner, cuestiones sobre la relación entre los diferentes actores en cuanto a: el trato que tienen los planners con diferentes participantes del entorno (cuentas, creatividad, agencias de medios, investigación, otras agencias del grupo) y si los planificadores que trabajan en agencias de publicidad reciben y comparten información sobre el consumidor con otros actores (agencias de medios, institutos de investigación, anunciante).

Por otro lado, en concreto se abunda en la relación del planner con el anunciante siguiendo estas variables: cuál es la fuente principal sobre el consumidor para los anunciantes; qué tipo de relación tienen los planners con los anunciantes; cuál es la aportación principal que los anunciantes esperan de la agencia; a quién interesa más la información sobre los consumidores; qué objetivos tienen; cuál es su ventaja competitiva y qué presupuesto destinan a medios convencionales y a no convencionales.

Para que se entienda mejor se van a desarrollar los resultados uno por uno y al final se realizará un resumen valorativo.

\section{a) Colaboración-competencia}

En primer lugar, se va a destacar qué tipo de relación tienen los planners con los diferentes participantes del entorno según una escala desde total colaboración a mucha competencia. Lo primero que llama la atención es que los planners manifiestan tener algo de competencia con las agencias de medios, con los institutos de mercado, con los anunciantes y con otras agencias del grupo. Por otro lado, la mayoría de los planners que pertenecen a agencias de publicidad destacan la total colaboración con Cuentas o Creatividad. 
¿Qué tipo de relación tienen los planners con diferentes actores del proceso comunicación comercial?

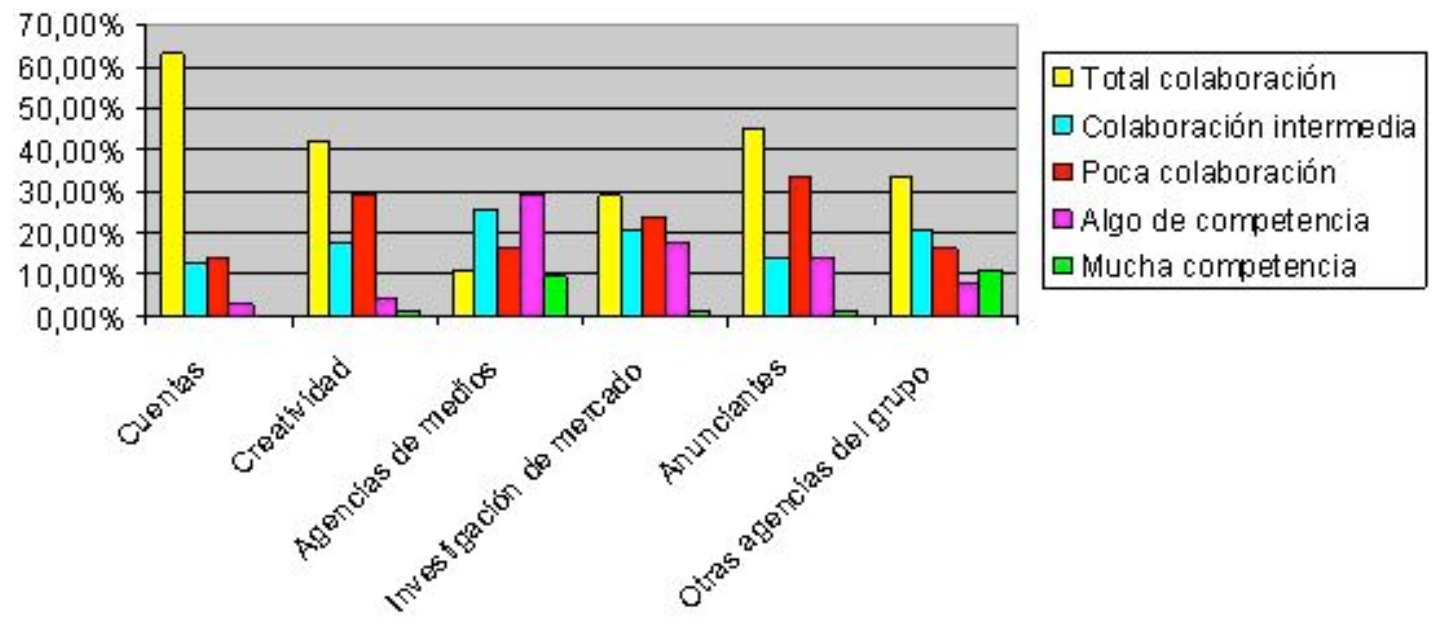

Gráfico 2 Elaboración propia con datos del estudio.

Una manifestación concreta de si hay colaboración o competencia es si reciben y comparten su conocimiento sobre el consumidor.

b) Si comparten y reciben información sobre el consumidor con otros actores

Para una gestión integrada del conocimiento del consumidor, lo ideal es que se reciba y se comparta la investigación entre todos los actores. Sin embargo, de las opciones que se les daba (siempre, bastantes veces, pocas veces y nunca) sobresale que los planners indican que comparten "pocas veces" en un $51,60 \%$ y "nunca" en un $25,80 \%$ con las agencias de medios y "pocas veces" en un $38,70 \%$ y "nunca" en un $24,20 \%$ con los institutos de investigación.

Sobre la recepción de información acerca de consumidor, llama la atención que los planners manifiestan que reciben "pocas veces" en un $50 \%$ de las agencias de medios y "pocas veces" en un $41,9 \%$ de los institutos de investigación.

c) Fuente de información sobre los consumidores para los anunciantes

La mayoría de los planners cree que los institutos de investigación son la fuente esencial de información sobre los consumidores para los anunciantes. Lo señala así el 69,4\%.

d) Tipo de relación del planner con los anunciantes

El $83,9 \%$ de los planners encuestados se relaciona directamente con los departamentos de marketing de los anunciantes. 
e) Aportación principal de la agencia a los anunciantes

Sobre la aportación principal que los anunciantes esperan de la agencia vemos que es la creatividad, seguida de la estrategia.

Aportación principal que los anunciantes esperan de la agencia

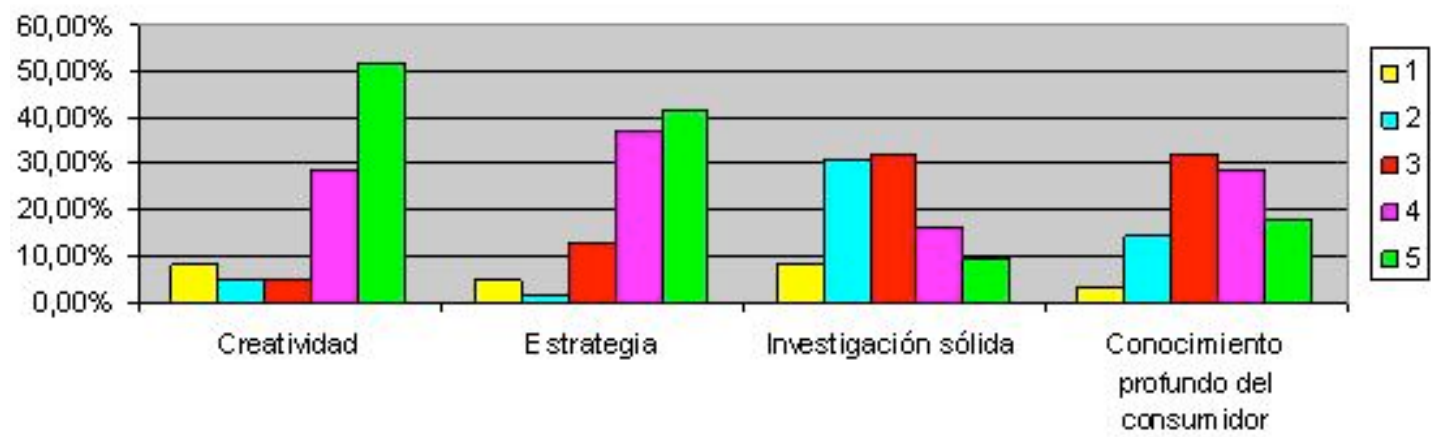

Gráfico 3. Elaboración propia con datos del estudio.

f) Interés sobre la información del consumidor: a creativos o a anunciantes

Al tener que optar entre los creativos (para orientar la ejecución de la campaña) o los anunciantes, los planners consideran que su conocimiento sobre el consumidor interesa más al anunciante, un $59,7 \%$, que a los creativos, un $37,1 \%$.

g) Objetivos de los anunciantes

Los anunciantes buscan sobre todo objetivos a corto plazo y en un 56,5\%, los planners, consideran que los anunciantes piensan "poco" en términos de comunicación integrada de marketing. Sobre si buscan información acerca del consumidor, vemos que el porcentaje es muy parecido en "bastante" y en "poco".

\section{Tipos de objetivos de los anunciantes}

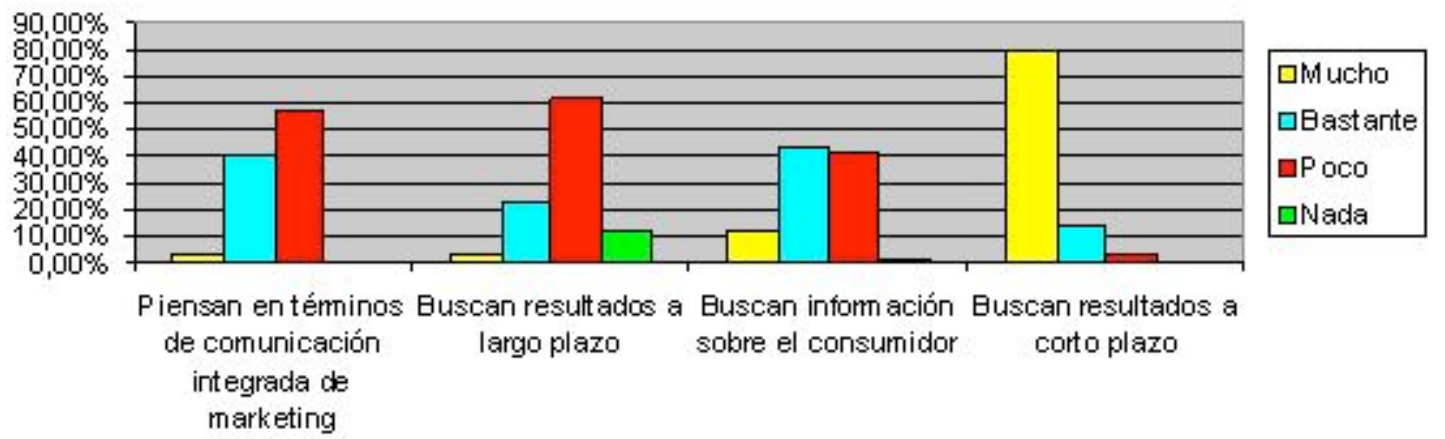

Gráfico 4. Elaboración propia con datos del estudio.

h) Ventaja competitiva de los anunciantes 
Según los planners, para los anunciantes la principal ventaja competitiva es, de igual modo, el desarrollo de productos y el conocimiento profundo del consumidor, ya que el porcentaje que corresponde a cada opción es el mismo, un 35,5\% en cada una.

i) Presupuesto destinado por los anunciantes a medios convencionales y a medios no convencionales

Como vemos, sigue siendo mayor el destinado a medios convencionales, a pesar de que la tendencia general del mercado es un crecimiento de la inversión en medios no convencionales.

Presupuesto destinado por los anunciantes

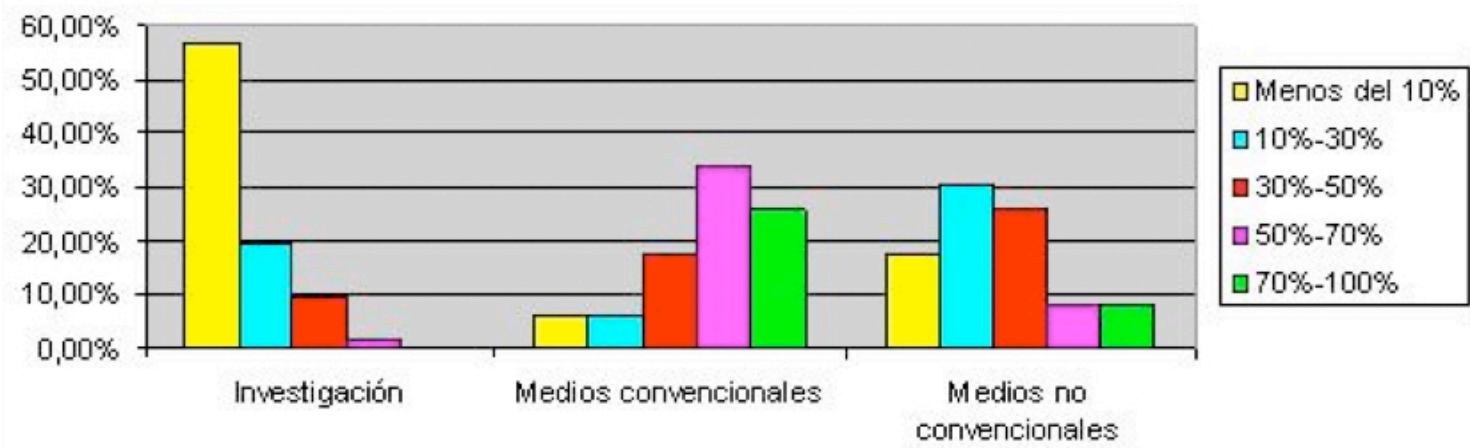

Gráfico 5. Elaboración propia con datos del estudio.

Resumiendo las ideas que hemos comentado en este apartado, en primer lugar, lo más reseñable es que no hay mucha colaboración entre los diferentes actores del entorno de comunicación comercial. Destacamos dos tipos de relaciones protagonizadas por las agencias de publicidad: hacia dentro (con los miembros de la propia agencia y con otras agencias del grupo) y hacia fuera (con las agencias de medios, con los anunciantes y con los institutos de investigación).

Por un lado, los planners manifiestan una total colaboración hacia dentro de las agencias de publicidad (con Cuentas y con Creatividad), con los anunciantes y con otras agencias del grupo. Pero, por otro, señalan un grado de competencia importante con las agencias de medios, los institutos de investigación y también con otras agencias del grupo. A esto se une la poca relación que tienen al no recibir ni compartir a penas entre sí información sobre el consumidor. Esto es importante porque, si desde la agencia se investiga al consumidor y también se hace desde la agencia de medios y los institutos de investigación, lo más efectivo es que ese conocimiento se comparta para que sea beneficioso para las decisiones del anunciante.

Los planners se relacionan directamente con los anunciantes y opinan que la aportación principal que estos esperan de la agencia es la creatividad y en segundo lugar la estrategia. 
No destacan el conocimiento en profundidad sobre el consumidor. Además, señalan que la fuente de información principal sobre los consumidores para los anunciantes son los institutos de investigación.

En esta misma línea, destacan que la principal ventaja competitiva de los anunciantes es, a la vez, el desarrollo de productos y el conocimiento profundo sobre el consumidor y matizan que los anunciantes no destinan mucho presupuesto a medios no convencionales, con los que podrían relacionarse más directamente y de manera más personalizada con los consumidores.

En síntesis, todavía es prematuro afirmar, desde el punto de vista de los planificadores estratégicos, que en España los profesionales de la comunicación comercial realizan una gestión integrada del conocimiento del consumidor, ya que no le posicionan en el centro de sus objetivos y de sus acciones y no trabajan de manera coordinada teniendo como nexo al consumidor.

\section{Conclusiones}

Hoy en día, la ventaja competitiva de las empresas tiene como núcleo la comprensión fundamental del consumidor. Ese conocimiento profundo debe ser el punto de partida en la planificación de una estrategia coherente para alcanzar la eficacia y el establecimiento de un compromiso a largo plazo entre empresa y consumidor.

Debido al contexto descrito, parece acreditada la necesidad de un especialista que gestione de manera integrada el conocimiento del consumidor. Hoy en día, el planificador estratégico, a través del diálogo continuo con el consumidor, es el mejor capacitado para saber dónde y a través de qué tipo de comunicación se le puede encontrar con eficacia. Por ello, participa de la estrategia de marca y de medios, realiza una investigación global sobre el consumidor y es la voz del mercado y el guardián de la marca.

En un contexto en el que el conocimiento del consumidor debe ser transversal, los anunciantes deben pensar más de forma integrada poniendo como centro de sus acciones al consumidor y no el desarrollo de productos $u$ otras variables que no permiten diferenciarse de la competencia a largo plazo.

Asimismo, es necesario que las empresas, junto con el resto de actores implicados en la comunicación comercial, trabajen en un entorno de colaboración y no de competencia, llevando a cabo una gestión integrada del conocimiento del consumidor que debe liderar el planificador estratégico.

Después de analizar la relación que tienen los planificadores estratégicos españoles con los demás actores del proceso de comunicación comercial, se puede destacar que no trabajan de una manera conjunta y unida para comprender y gestionar el conocimiento del consumidor. 
En definitiva, se debe seguir trabajando en la investigación, en el desarrollo de modelos de gestión del consumidor y en el análisis a fondo de las posibilidades que presenta la Planificación Estratégica para posicionar al consumidor como eje de la comunicación comercial. 


\section{REFERENCIAS BIBLIOGRÁFICAS}

ARENS, William F., Schaefer, David H. (2007): Essentials of contemporary advertising. NY, Mc Graw-Hill/Irwin.

BASKIN, Merry, PICKTON, David (2003): "Account planning, from genesis to revelation", en Marketing Intelligence and Planning, 21, 7, pp. 416-424.

CALDER, B.J. (2001): "Understanding consumers", en IACOBUCCI, Dawm (Ed.), Kellogg on marketing: the Kellogg Marketing Faculty Northwestern University, New York, Wiley \& Sons, pp. 163-164.

CHIESA, Cosimo (2002): Fidelizando para fidelizar. Cómo dirigir, organizar y retener a nuestro Equipo Comercial. Pamplona, Eunsa.

CHIESA, Cosimo (2005): CRM: las cinco pirámides del Marketing Relacional. Barcelona, Ediciones Deusto.

Considine, P. (2007): "Understanding the digital consumer", en Campaign 13 April, pp. 24-26.

Duncan, Tom (2005): Principles of Advertising \& IMC, New York, Mc Graw-Hill.

FAUCONNIER, C. (2006): "Humanising the marketplace: a manifesto for brand growth", en Admap, April, pp. 35-37.

FERNÁNDEZ NOGALES, A. (1999): Investigación de mercados: obtención de información. Madrid, Civitas.

Fleming, Paul, alberdi, María José (2000): Hablemos de marketing interactivo. Reflexiones sobre marketing digital y comercio electrónico. Madrid, ESIC Editorial.

FRANZ, G. (2000): "Better media planning for integrated communication", en Admap, January, pp. 41-42.

GARCíA, César (2007): El libro de Bob. La nueva publicidad del siglo XXI. Madrid, Zapping/ M\&C Saatchi.

Godin, Seth (1999): Permission Marketing. Turning strangers into friends and friends into customers. New York, Simon \& Schuster.

GRANT, John (2000): The new marketing manifesto. The 12 rules for building successful brands in the 21st century. New York, Thomson Texere.

HumBY, C. (2004): "Is consumer marketing irrevocably broken?", en Admap, October.

JONES, R.W. (1968): "Are media departments out of date?", en Admap, September.

KAYE, T. (2002): "Media-neutral planning -just today's buzzword or a genuine new world order?",

en www.apg.org.uk/publications/medianeutralarticles/mnp1_kayetina.cfm (consultado: 10/10/08). 
Kelley, Larry D., JUgenheimer, Donald W. (2006): Advertising account planning. A practical guide. New York, M.E. Sharpe.

KITCHEN, Philip, SCHULtZ, Don E. (2000): "The status of IMC: a 21st-century perspective", en Admap, September, pp. 18-19.

o 'MALLeY, Damian (1999): "Account Planning: an American perspective", en JONES, John Philipp (Ed.): The advertising business: operations, creativity, media planning and integrated communications. California, Sage Publications.

OlLÉ, Ramón (2005): "El Planner: la voz del consumidor en la agencia", en FERNÁNDEZ GÓMEZ, Jorge David (Coord.): Aproximaciones a la estructura de la publicidad. Desarrollo y funciones de la actividad publicitaria. Sevilla, Comunicación Social Ediciones y Publicaciones, pp. 115-131.

PeDRET, Ramón, SAGNieR, Laura, CAMP, Fransesc (2000): La investigación comercial como soporte del marketing. Barcelona, Deusto.

PÉREZ LATRE, Francisco Javier (2007): "The paradigm shift in advertising and its meaning for advertising-supported media", en Journal of Media Business Studies, 4, 1, Spring, pp. 41-49.

RAPP, Sthan, COLLINS, Thomas (1991): El nuevo rumbo del marketing. Un gran giro en la concepción del mercado. Madrid, McGraw-Hill/Interamericana de España S.A.

RENSMANN, F.J. (2000): "Customer ownership: business planning through customers", en Admap, December, pp. 17-18.

SÁNCHEZ BLANCO, Cristina (2009): "Génesis de la Planificación de cuentas: cómo gestionar el conocimiento del consumidor en las agencias de publicidad", Comunicación y Sociedad, XXII, 2, pp. 187-219.

Schultz, Don E., tannenbaum, Stanley, LaUterborn, Robert (1994): Integrated marketing communications. Lincolnwood Chicago, NTC Business Books.

SChultz, Don E., tannenbaum, Stanley, LAUTERBoRn, Robert (1996): The new marketing paradigm. Integrated marketing communications. Lincolnwood Chicago, NTC Business Books.

SiRkIN, Karen (2004): "The future of communications planning", en Admap, October, pp. 87-88.

SWAIN, William N. (2004): "Perceptions of IMC after a decade of development: who's at the wheel and how can we measure success?", en Journal of Advertising Research, March, pp. 46-65.

VAN RAAIJ, W.F. (1998): "Interactive communication: consumer power and initiative", en Journal of Marketing Communication, 4, 1-8. 
Vollmer, Christopher, PRECOURT, Geoffrey (2008): Always on. Advertising, marketing and media in an era of consumer control. New York, McGraw Hill.

YESHIN, Tim, (1998): Integrated Marketing Communications: the holistic approach. Oxford, Butterworth-Heinemann.

Zaltman, G. (2004): Cómo piensan los consumidores. Lo que nuestros clientes no pueden decirnos y nuestros competidores no saben. Madrid, Ediciones Urano.

ZAMBARDiNo, Adrian, GOODFELlow, Jon (2003): "Account planning in the new marketing and communications environment (has the Stephen King challenge been met?)", en Marketing Intelligence and Planning, 21,7, pp. 425-434.

http://www.anuncios.com/inversion-publicitaria/masanuncios/1028854009501/media-planning-mccann-erickson.1.html (consultado: 29/09/08).

[Recibido: 13 de octubre de 2010. Aceptado (con indicación de cambios): 8 de julio de 2011 . Recepción del artículo corregido: 19 de julio de 2011.] 